\title{
Energy balance-related behavioural patterns in 5-year-old children and the longitudinal association with weight status development in early childhood
}

\author{
Jessica S Gubbels ${ }^{1, *}$, Stef PJ Kremers ${ }^{1}$, R Alexandra Goldbohm² ${ }^{2}$ Annette Stafleu ${ }^{2}$ and \\ Carel Thiis ${ }^{3}$ \\ 'Department of Health Promotion, Maastricht University, NUTRIM School for Nutrition, Toxicology and \\ Metabolism, PO Box 616, 6200 MD Maastricht, The Netherlands: ${ }^{2}$ TNO, Leiden/Zeist, The Netherlands: \\ ${ }^{3}$ Department of Epidemiology, Maastricht University, School for Public Health and Primary Care (CAPHRI), \\ Maastricht, The Netherlands
}

Submitted 4 July 2011: Accepted 25 October 2011: First published online 29 November 2011

\begin{abstract}
Objective: The current study examined clustering of dietary intake and activity behaviours (i.e. physical activity (PA) and sedentary behaviour) in 5-year-old children, as well as the longitudinal association with BMI and overweight development.

Design: Principal component analysis (PCA) was used to identify behavioural patterns. Backward regression analyses were used to examine the association of behavioural patterns with parent and child background characteristics, as well as the association of the patterns with BMI Z-score and overweight development up to ages 7 and 8 years.

Setting: The Netherlands.

Subjects: Data originated from the KOALA Birth Cohort Study ( $n 2074$ at age 5 years). Results: Four patterns emerged: a 'sedentary-snacking' pattern (including television viewing and snack consumption), a 'healthy intake' pattern (including healthy items such as fruit, vegetables and fish), a 'sandwich' pattern (including bread and sandwich fillings) and a 'sporty-traditional meal' pattern (including sports, meat and potatoes). The patterns were related to child gender, general appetite and various parental characteristics. The sedentary-snacking pattern was positively associated with longitudinal BMI development.

Conclusions: Cross-behavioural energy balance-related behavioural patterns exist at age 5 years. Different activity types (e.g. sports, television viewing) clustered differently with the behavioural patterns, indicating the need to incorporate these different types instead of one general PA measure in energy balance-related pattern analyses. The clustering and potential synergy between activity behaviours and dietary intake, as well as the associations with weight status development, stress the importance of an integrated approach to PA and healthy nutrition promotion in preventing childhood overweight.
\end{abstract}

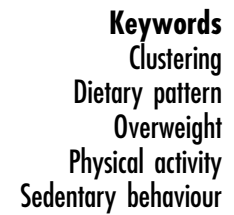

Clustering

Overweight Sedentary behaviour
Overweight and obesity are a rapidly growing problem, affecting not only adults but children worldwide as well $^{(1,2)}$. Furthermore, overweight children often become overweight or obese adults ${ }^{(3)}$. The most important determinants of childhood overweight and obesity include the consumption of energy-dense foods, frequent television viewing and computer use, and low levels of physical activity $(\mathrm{PA})^{(4)}$. Several studies have examined the co-occurrence, or 'clustering', of such 'energy balancerelated behaviours' (see below for examples). Clustering is defined as a combination of behaviours which is more prevalent than expected based on the prevalence of the separate behaviours ${ }^{(5)}$. The potential synergy between energy balance-related behaviours could be used in obesity prevention interventions, applying an integrated approach addressing multiple behaviours simultaneously ${ }^{(6)}$.

Identifying behavioural patterns in young children is important, since dietary and PA habits are formed at early life stages and track into later life ${ }^{(7,8)}$. Moreover, childhood dietary patterns have been shown to track into adulthood $^{(9,10)}$. Principal component analysis (PCA) and cluster analysis are both frequently used and validated methods for examining the existence of dietary patterns ${ }^{(11,12)}$. Numerous studies have applied one of these techniques to examine either dietary patterns ${ }^{(9,10,13-19)}$ or activity patterns ${ }^{(20,21)}$ in children. Fewer studies have looked into cross-behavioural 
clustering (i.e. clustering between PA and dietary intake) in children. These studies show some comparable behavioural patterns, including a 'sedentary-snacking' pattern, in which intake of unhealthy food items (e.g. snacks, soft drinks) clusters with sedentary behaviour (i.e. television and computer use $)^{(22-28)}$. In addition, previous studies often report an 'all-round-healthy' pattern of healthy food intake and high levels of $\mathrm{PA}^{(23-26,28,29)}$ and/or low levels of sedentary behaviour $^{(24,28)}$. However, in contrast to the food groups used in behavioural pattern analyses, which are often assessed thoroughly using validated FFQ or other detailed instruments, activity behaviours are often assessed quite poorly or aggregated into one or two measures summarizing all activity behaviours. All cross-behavioural clustering studies described above were limited to only one measure of PA (e.g. minutes of exercise ${ }^{(25)}$ ), except for one study that differentiated between moderate and vigorous $\mathrm{PA}^{(29)}$.

Energy balance-related behavioural patterns have been found to be associated with various background characteristics of the child and parents. For instance, girls were more likely to have a healthy intake pattern ${ }^{(27)}$, while boys were more likely to have a sporty-healthy eating pattern ${ }^{(29)}$ or a high active/high sedentary behavioural pattern $^{(27)}$. A lower parental educational level has been found to be positively associated with unhealthy behavioural patterns, and negatively with healthy patterns ${ }^{(22)}$. Maternal obesity $^{(22)}$ and lower socio-economic status ${ }^{(23)}$ predicted higher scores on the unhealthy sedentary-snacking pattern.

Various behavioural patterns have previously been linked to overweight-related measures. A healthy behavioural pattern (high levels of PA and/or healthy diet) was inversely associated with overweight risk ${ }^{(23,25)}$, while the sedentary-snacking pattern was positively associated with overweight $^{(23)}$. However, other studies find no association with BMI or overweight ${ }^{(22,24,27,29)}$, possibly due to the crosssectional design of those studies.

The current study examined clustering of energy balancerelated behaviours of 5-year-olds. In line with previous findings we hypothesized to find patterns similar to the general patterns described above (e.g. a healthy pattern, a sedentary-snacking pattern). The current study adds to previous research by using detailed behavioural measures for both dietary intake and activity behaviours. We further examined the association of the identified patterns with background variables of the child (e.g. gender) and parents (e.g. educational level). Finally, we examined the longitudinal association of the dietary patterns with BMI and weight status.

\section{Methods}

\section{Respondents and procedure}

The KOALA Birth Cohort Study (The Netherlands) is a prospective cohort study which started in 2000. Healthy pregnant women were recruited from an existing cohort, as well as through 'alternative lifestyle' recruitment channels (e.g. anthroposophist midwives, organic food shops) ${ }^{(30)}$. The latter group (17.9\%) could have an alternative lifestyle with regard to dietary habits (e.g. organic, vegetarian), child rearing, vaccination schemes or antibiotics use. All participants signed informed consent, and ethical approval was obtained from the Maastricht University/ University Hospital Maastricht medical ethics committee. A total of 2834 women completed questionnaires during pregnancy and after birth.

\section{Questionnaire}

When the children reached the age of about 5 years old, parents completed an extensive questionnaire regarding various background characteristics, their children's dietary intake, activity behaviours and BMI, and other relevant factors ( $n$ 2074; 73.2\%). Follow-up questionnaires, assessing only child BMI, were sent around ages 7 years ( $n$ 2002; $96 \cdot 5 \%)$ and 8 years ( $n 1828 ; 88 \cdot 1 \%$ ). The questionnaires are described in more detail below.

\section{Child activity behaviour}

Children's activity behaviour was assessed using the Standard Questionnaire for measuring Physical Activity, used in Dutch Youth Health Care ${ }^{(31)}$. Parents were asked on how many days in a normal week during the last 4 weeks their child had gone to school on foot or by bicycle, played sports at school (e.g. during physical education lessons), played sports at a sports club and played outside (outside school hours). A second question assessed the average duration of each activity. The duration and number of days were multiplied to calculate the total number of minutes spent on each activity per week. Sedentary screenbased behaviour was assessed in a similar manner, asking for television watching (including videos and DVDs) and computer playing. Table 1 provides an overview of the activity behaviours assessed.

\section{Child dietary intake}

Children's dietary intake was assessed using a detailed FFQ, developed to assess children's energy intake and validated using the doubly labelled water method (Pearson correlation coefficient $=0 \cdot 62)^{(32)}$. Parents indicated their child's average consumption frequency during the 4 weeks preceding the questionnaire, by checking one of six frequency categories ranging from 'never' to '6-7 d/week'. Respondents were asked to report portion sizes in natural units (e.g. pieces, slices), household units (e.g. glasses, spoons) or grams. Intake frequency and portion size were then multiplied to obtain average intake.

The intake of the foods was categorized into twentyfour food groups (Table 1). The energy intake (in kilojoules) per week from each group was calculated using the 2001 Netherlands Food Composition (NEVO) table ${ }^{(33)}$. The energy intake was calculated per week in order to facilitate comparison with the activity behaviours. 
Nutritional values of products that were not included in the 2001 NEVO table were estimated by a dietitian.

\section{Child BMI and weight status}

Parents were asked in the 5-year questionnaire to report their child's weight and height (measured without shoes and clothes, specified to one decimal), in order to calculate the child's BMI (i.e. weight $(\mathrm{kg}) /[\text { height }(\mathrm{m})]^{2}$ ). BMI was then recoded into age- and gender-specific BMI $Z$-scores compared with the national reference population $^{(34)}$. Parents were asked to indicate their child's weight and height again in follow-up questionnaires at ages 7 and 8 years, in order to calculate BMI $Z$-scores for those ages. A BMI $Z$-score $\geq 85$ th percentile was considered overweight $^{(35)}$.

\section{Child and parental background factors}

Child gender was assessed in a previous questionnaire. In the 5-year questionnaire, parents were asked to indicate their child's general appetite and activity style, using two items each. For general appetite, parents were asked to indicate on a scale from 1 ('completely disagree') to 5 ('completely agree') to what extent the following statements applied to their child: 'Compared to peers, my child is always hungry' and 'Compared to peers, my child has a small appetite'. The latter item was reverse coded, after which the two items were averaged to get a score from 1 to 5 for the child's general appetite. The activity style was assessed in a similar way using the items 'Compared to peers, my child is very active' and 'Compared to peers, my child never sits still'.

Various parental background factors were assessed, including the number of hours per week that fathers and mothers worked, their highest completed educational level and their country of birth. Educational level was recoded into three levels (low, medium, high) ${ }^{(36)}$. Country of birth was recoded into 'Netherlands' $v$. 'other'. Parents were further asked to indicate their own weight and height, in order to calculate parental BMI (in $\mathrm{kg} / \mathrm{m}^{2}$ ).

\section{Analyses}

The analyses were conducted using the SPSS statistical software package version $15 \cdot 0$ (SPSS Inc., Chicago, IL, USA). $P$ values $<0 \cdot 05$ were considered statistically significant.

PCA with oblique rotation was performed to examine behavioural patterns ${ }^{(37)}$. All variables were standardized, and a scree plot was used to determine the number of components $^{(37)}$. Behavioural items with absolute component loadings $>0.4$ were considered part of the pattern $^{(38)}$. Behavioural pattern scores were calculated using the regression method ${ }^{(37)}$.

Given the explorative nature of the analyses ${ }^{(37)}$, backward linear regression analyses were conducted to examine the association between various background characteristics and the pattern scores. The background characteristics included in the analyses were child gender,
BMI Z-score at age 5 years, general appetite and activity style, and maternal and paternal working hours, educational level, country of birth and BMI. Dummy variables for high and low compared with medium educational level were created for the regression analyses. The analyses were further adjusted for recruitment group (alternative $v$. conventional lifestyle).

Separate backward linear and logistic regression analyses were conducted to examine the association of each of the pattern scores with children's BMI and weight status at the follow-up points at age 7 and 8 years, corrected for BMI or weight status at age 5 years, respectively. For this purpose, the children were divided into tertiles for each pattern. The dummy variables for each tertile were included as independent variables in the analyses. These analyses were corrected for various background characteristics: child gender, BMI $Z$-score at age 5 years, general appetite and activity style, parental working hours, educational level, country of birth and BMI, and recruitment group. As these analyses were corrected for BMI $Z$-score or weight status at age 5 years, the outcomes reflect development of BMI and weight status between ages 5 and 7 years, and between ages 5 and 8 years, respectively.

\section{Results}

In total, $51 \cdot 3 \%$ of the children participating at age 5 years were male. Mean birth weight was 3524 (SD 508) g, mean BMI $Z$-score at age 5 years was $-0 \cdot 27$ (SD 0.99). Fathers worked for an average of $37 \cdot 8$ (SD 10.1) h/week, while mothers worked for $17 \cdot 9$ (SD 11.1) h/week. More than half of the mothers $(54 \cdot 2 \%)$ and fathers $(53 \cdot 4 \%)$ had a high educational level, while only $8.0 \%$ of the mothers and $12 \cdot 8 \%$ of the fathers had a low educational level. Almost all parents $(97 \cdot 0 \%$ of the mothers, $96 \cdot 3 \%$ of the fathers) were born in The Netherlands. Mean maternal BMI was $24 \cdot 0$ (SD $3 \cdot 8$ ) $\mathrm{kg} / \mathrm{m}^{2}$, mean paternal BMI was $25 \cdot 0$ (sD $3 \cdot 1) \mathrm{kg} / \mathrm{m}^{2}$.

\section{Child behaviour}

Table 1 provides an overview of the averages of the activity and dietary intake behaviours. Most time was spent playing outside: almost $1.5 \mathrm{~h} / \mathrm{d}$ (623 min/week) on average. Other PA behaviours were performed far less. The total average time spent being physically active was 795 (SD 378) $\mathrm{min} /$ week. The total sedentary time (i.e. television and computer use) was 411 (SD 293) $\mathrm{min} /$ week.

The average total energy intake of the children was 43210 (sD 8994) kJ/week. Children consumed by far most of their energy from the 'Bread and breakfast products' (7267 kJ/week) and 'Dairy' (6829 kJ/week) groups. Other food groups that contributed substantially to children's energy intake were 'Pastry and cookies', 'Sugar-sweetened drinks', 'Meat and meat substitutes' and 'Composite dishes'. 
Table 1 Energy balance-related behavioural categories and their descriptive statistics among the study population ( $n$ 2074): KOALA Birth Cohort Study, The Netherlands

\begin{tabular}{|c|c|c|c|}
\hline Behaviour & Content & Mean & SD \\
\hline \multicolumn{4}{|l|}{ Activity behaviourt (min/week) } \\
\hline Active transport & Walking or cycling to school & 46 & 55 \\
\hline School sports & $\begin{array}{l}\text { Sports at school, including physical education and swimming lessons as part of the } \\
\text { curriculum }\end{array}$ & 84 & 63 \\
\hline Sports at a club & Sports at a sports club (e.g. swimming, soccer, ballet) & 43 & 53 \\
\hline Playing outside & Playing outside, excluding school recess & 623 & 368 \\
\hline Television watching & Television watching, including videos and DVDs & 358 & 248 \\
\hline Computer use & $\begin{array}{l}\text { Using the computer, including using the Internet and playing games on a regular or } \\
\text { games computer }\end{array}$ & 53 & 103 \\
\hline \multicolumn{4}{|c|}{ 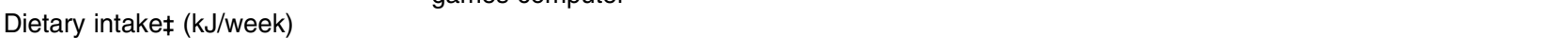 } \\
\hline Potatoes & Potatoes, fries & 1786 & 1087 \\
\hline Rice and pasta & Rice, pasta & 723 & 709 \\
\hline Meat and meat substitutes & Meat, meat products, poultry and meat substitutes & 3798 & 1918 \\
\hline Fish and seafood & Fish, crustaceans, shellfish & 532 & 544 \\
\hline Sauce & Mayonnaise, gravy, tomato sauce & 518 & 465 \\
\hline Composite dishes & Pancakes, pizza, noodles, lasagne, quiche & 2278 & 1562 \\
\hline Legumes & Legumes (e.g. beans) & 94 & 190 \\
\hline Vegetables & Cooked and raw vegetables & 403 & 241 \\
\hline Fruit & Fresh fruits & 1998 & 1018 \\
\hline Bread and breakfast products & Bread, cold and hot cereal, crackers & 7267 & 2242 \\
\hline Savoury sandwich fillings & Peanut butter & 763 & 1163 \\
\hline Sweet sandwich fillings & Jam, chocolate sprinkles, chocolate spread, syrup on bread & 1811 & 1443 \\
\hline Cheese & Soft and hard cheese & 1225 & 1119 \\
\hline Eggs & Eggs & 345 & 314 \\
\hline Fats & Butter, margarine, low-fat margarine & 1712 & 1041 \\
\hline Dairy & $\begin{array}{l}\text { Milk, chocolate milk, milk drinks, yoghurt, yoghurt drinks, cottage cheese, } \\
\text { milk porridge, custard, whipping cream }\end{array}$ & 6829 & 3893 \\
\hline Soya products & Soya milk, soya desserts & 317 & 1606 \\
\hline Nuts & Nuts, peanuts & 103 & 348 \\
\hline Sweets and sugar & $\begin{array}{l}\text { Ice cream, chocolate, chewing gum, sweets, candy bars, liquorice, raisins, sugar, } \\
\text { honey, syrup }\end{array}$ & 1941 & 1150 \\
\hline Pastry and cookies & Biscuits, cake, pie, waffles, granola bars, bread sticks, rice crackers & 3400 & 1817 \\
\hline Savoury snacks & Potato chips, savoury biscuits, fried meat snacks & 1255 & 977 \\
\hline Fruit juice & Fruit juice, fruit drinks & 1230 & 1718 \\
\hline Sugar-free or diet drinks & Water, tea, diet soft drinks & 3 & 7 \\
\hline Sugar-sweetened drinks & Sugar-sweetened soft drinks, sugar-sweetened drinking syrup & 2880 & 2694 \\
\hline
\end{tabular}

†Average total physical activity = 795 (SD 378) $\mathrm{min} /$ week, average sedentary time $=411$ (SD 293) $\mathrm{min} / \mathrm{week}$.

$\ddagger$ Average total energy intake $=43210$ (sD 8994) kJ/week.

\section{Chustering of child bebaviour: behavioural patterns}

Based on a scree plot, the PCA revealed four patterns. The component loadings of each behaviour for each of these patterns can be found in Table 2. The first pattern, named the 'sedentary-snacking pattern', was characterized by much television watching, and high intakes of sweets and sugar, pastry and cookies, savoury snacks and sauce. A second pattern included high intake of rice and pasta, fish and seafood, legumes, vegetables and fruit, and was called the 'healthy intake pattern'. The third pattern comprised high intakes of bread and breakfast products, sweet sandwich fillings and fats, the 'sandwich pattern'. A fourth pattern included sports at school and at the sports club, as well as intakes of potatoes, meat and meat substitutes, and was characterized as the 'sportytraditional meal pattern'. The four patterns explained $26.1 \%$ of the variation in the energy balance-related behaviours, and showed mutual correlations ranging from 0.007 (NS) between the sedentary-snacking pattern and the healthy intake pattern, up to $-0 \cdot 161(P<0 \cdot 001)$ between the healthy intake pattern and the sporty-traditional meal pattern.

\section{Associations of background characteristics with bebavioural patterns}

Various child and parental background characteristics were related to the pattern scores (Table 3). Girls scored lower than boys on the sedentary-snacking pattern and the sandwich pattern. A large general appetite was associated with higher scores on the healthy intake and sandwich patterns.

Higher parental BMI was related with higher scores on the sporty-traditional meal pattern. Higher maternal BMI was further associated with lower scores on the healthy intake pattern, while higher paternal BMI was associated with higher scores on the sedentary-snacking pattern and lower scores on the sandwich pattern. Parents' educational level was inversely related with scores on the sedentarysnacking pattern. Maternal educational level was further positively associated with the sandwich pattern and higher paternal educational level was positively related with the 
Table 2 Component loadings of principal component analysis on energy balance-related behaviour among the study population: KOALA Birth Cohort Study, The Netherlands

\begin{tabular}{|c|c|c|c|c|}
\hline & \multicolumn{4}{|c|}{ Pattern } \\
\hline & 1 & 2 & 3 & 4 \\
\hline \multicolumn{5}{|l|}{ Activity behaviourt } \\
\hline Active transport & -0.059 & 0.059 & 0.078 & $0 \cdot 246$ \\
\hline School sports & -0.021 & 0.041 & -0.006 & $0 \cdot 413$ \\
\hline Sports at a club & -0.011 & 0.029 & $-0 \cdot 010$ & 0.511 \\
\hline Playing outside & 0.229 & 0.116 & 0.025 & $5-0.255$ \\
\hline Television watching & 0.429 & $-0 \cdot 198$ & -0.087 & $7 \quad 0.271$ \\
\hline Computer use & 0.282 & $-0 \cdot 127$ & 0.060 & 0.288 \\
\hline \multicolumn{5}{|l|}{ Dietary intakeł } \\
\hline Potatoes & 0.393 & $0 \cdot 318$ & -0.007 & 0.420 \\
\hline Rice and pasta & -0.036 & 0.589 & 0.059 & $9-0.160$ \\
\hline Meat and meat substitutes & 0.251 & 0.238 & 0.069 & 9.566 \\
\hline Fish and seafood & 0.023 & 0.453 & -0.040 & $0 \cdot 228$ \\
\hline Sauce & 0.491 & 0.077 & 0.016 & $0 \cdot 118$ \\
\hline Composite dishes & 0.354 & $0 \cdot 227$ & $0 \cdot 102$ & -0.133 \\
\hline Legumes & -0.012 & 0.409 & $-0 \cdot 107$ & $7 \quad 0.030$ \\
\hline Vegetables & -0.041 & 0.754 & 0.021 & $0 \cdot 124$ \\
\hline Fruit & $-0 \cdot 114$ & 0.428 & 0.069 & -0.027 \\
\hline Bread and breakfast products & -0.015 & 0.076 & 0.830 & 0.082 \\
\hline Savoury sandwich fillings & 0.083 & $0 \cdot 107$ & $0 \cdot 174$ & $4-0.307$ \\
\hline Sweet sandwich fillings & 0.055 & -0.220 & 0.590 & 0.147 \\
\hline Cheese & -0.049 & 0.336 & $0 \cdot 294$ & $7-0.149$ \\
\hline Eggs & 0.275 & $0 \cdot 276$ & 0.016 & -0.136 \\
\hline Fats & -0.001 & 0.007 & 0.728 & $3-0.077$ \\
\hline Dairy & 0.263 & -0.115 & 0.075 & $5 \quad 0.010$ \\
\hline Soya products & -0.093 & 0.078 & -0.034 & $4-0.010$ \\
\hline Nuts & $0 \cdot 135$ & $0 \cdot 165$ & -0.015 & -0.323 \\
\hline Sweets and sugar & 0.656 & 0.001 & -0.048 & $3-0.176$ \\
\hline Pastry and cookies & 0.510 & -0.026 & -0.019 & $9 \quad 0.002$ \\
\hline Savoury snacks & 0.510 & $-0 \cdot 127$ & -0.076 & 0.273 \\
\hline Fruit juice & 0.237 & 0.058 & $-0 \cdot 124$ & $7-0 \cdot 100$ \\
\hline Sugar-free or diet drinks & $0 \cdot 135$ & -0.057 & -0.044 & $4 \quad 0.203$ \\
\hline Sugar-sweetened drinks & $0 \cdot 129$ & -0.015 & 0.015 & $5-0.025$ \\
\hline Variance explained (\%)§ & $8 \cdot 4$ & $7 \cdot 5$ & $5 \cdot 6$ & $4 \cdot 5$ \\
\hline
\end{tabular}

Results of oblique principal component analysis. Bold component loadings are $>0.4$ and are thus considered part of the pattern.

Pattern 1 = 'sedentary-snacking pattern'; pattern 2 = 'healthy intake pattern'; pattern $3=$ 'sandwich pattern'; pattern $4=$ 'sporty-traditional meal pattern'.

tActivity behaviours are measured in $\mathrm{min} /$ week.

‡Dietary intake is measured in $\mathrm{kJ} /$ week.

$\S$ Total variance in the behavioural variables explained by the four patterns is $26 \cdot 1 \%$.

healthy intake pattern and negatively with the sportytraditional meal pattern. The more hours mothers worked, the lower the scores on the sandwich and sporty-traditional meal patterns. Children of mothers who were not born in The Netherlands scored lower on the sporty-traditional meal pattern.

\section{Association of behavioural patterns with BMI and weight status development}

All analyses with BMI or weight status at follow-up points as a dependent variable were corrected for BMI and weight status respectively at age 5 years, as well for background variables. The results can be found in Table 4. The sedentary-snacking pattern was positively related to children's BMI at age 7 years ( $n$ 2002), as well as at 8 years ( $n$ 1828). Children in the highest tertile of the sedentary-snacking pattern had a significantly higher BMI Z-score at age 7 years (standardized regression coefficient, $\beta=0 \cdot 110, P<0 \cdot 001)$ than those in the lowest and middle tertiles. Also at age 8 years, the BMI $Z$-score in the highest tertile of the sedentary-snacking pattern was higher $(\beta=0.089, P<0 \cdot 001)$ compared with the lowest and middle tertiles. With regard to weight status, children in the highest tertile of the sedentary-snack pattern were also significantly more often overweight at age 7 years ( $\mathrm{OR}=2 \cdot 222, P<0 \cdot 01)$. None of the other patterns was significantly related to the children's longitudinal BMI or overweight development.

\section{Discussion}

The current study examined energy balance-related behavioural patterns in 5-year-old Dutch children, as well as the longitudinal association with overweight and BMI development. The study is the first to structurally assess and incorporate different types of physical activity (i.e. active transport, school sports, sports at a sports club, and playing outside) in the cross-behavioural pattern analyses of young children's energy balance-related behaviours, together with separate sedentary behaviours and a detailed selection of food groups. We found that these different types of physical activity indeed clustered differently with the various behavioural patterns. Despite the fact that some activity behaviours did not cluster with any of the behavioural patterns, our findings do stress the importance of differentiation of PA types in energy balance-related behaviour clustering studies, instead of incorporating one general PA measure. These findings are in line with a study of Jago and colleagues ${ }^{(20)}$, who examined clustering within activity behaviour (but not with dietary intake behaviour), and also found that different sedentary behaviours and PA types clustered within different behavioural patterns.

The current study identified four behavioural patterns, two of which were similar to previously found patterns: the sedentary-snack pattern and the healthy intake pattern. The sedentary-snack pattern included television watching and intake of relatively unhealthy sweet and savoury snacks. This is a very common pattern in children, encountered in many studies ${ }^{(22-28)}$. In addition, various of the studies that only included dietary intake items reported similar clustering of high intakes of snacks and other unhealthy energy-dense foods ${ }^{(10,13-16,18,19)}$. Apparently, this sedentary-snacking pattern is a universal behavioural pattern, found in developed countries all over the world (i.e. Europe, Asia, Australia, USA). Various plausible mechanisms behind the association between television watching and snacking have previously been proposed, including the stimulating influence of snack commercials $^{(39)}$, the provision of a context during sedentary activities that promotes passive snacking or overeating $^{(40)}$, and the distracting influence of television watching while eating, disrupting habituation to food cues (e.g. satiety) ${ }^{(41)}$. High levels of sedentary behaviour 
Table 3 Association of child and parental background variables with pattern scores: KOALA Birth Cohort Study, The Netherlands

\begin{tabular}{|c|c|c|c|c|c|c|c|c|}
\hline & \multicolumn{2}{|c|}{ Pattern 1} & \multicolumn{2}{|c|}{ Pattern 2} & \multicolumn{2}{|c|}{ Pattern 3} & \multicolumn{2}{|c|}{ Pattern 4} \\
\hline & $\beta$ & $95 \% \mathrm{Cl}$ & $\beta$ & $95 \% \mathrm{Cl}$ & $\beta$ & $95 \% \mathrm{Cl}$ & $\beta$ & $95 \% \mathrm{Cl}$ \\
\hline \multicolumn{9}{|l|}{ Child } \\
\hline \multicolumn{9}{|l|}{ Gender } \\
\hline Female $v$. male & $-0 \cdot 13^{\star \star \star}$ & $-0.18,-0.09$ & $t$ & & $-0 \cdot 18^{\star \star \star}$ & $-0.23,-0.14$ & $t$ & \\
\hline General appetite & $t$ & & $0 \cdot 24^{\star \star *}$ & $0 \cdot 20,0 \cdot 28$ & $0 \cdot 16^{\star * \star}$ & $0 \cdot 12,0 \cdot 20$ & $t$ & \\
\hline \multicolumn{9}{|l|}{ Maternal } \\
\hline \multicolumn{9}{|l|}{ Educational level } \\
\hline High $v$. medium & $-0 \cdot 08^{\star *}$ & $-0.13,-0.03$ & $t$ & & $0 \cdot 11^{\star \star *}$ & $0.06,0.15$ & $t$ & \\
\hline Low v. medium & $0 \cdot 08^{\star *}$ & $0 \cdot 03,0.13$ & t & & -0.04 & $-0.09,0.00$ & $t$ & \\
\hline \multicolumn{9}{|l|}{ Work } \\
\hline Hours per week & $t$ & & $t$ & & $-0 \cdot 06^{\star \star}$ & $-0.10,-0.02$ & $-0 \cdot 06^{\star \star}$ & $-0.10,-0.01$ \\
\hline \multicolumn{9}{|l|}{ Country of birth } \\
\hline Other $v . \mathrm{NL}$ & $t$ & & + & & $t$ & & $-0 \cdot 06^{\star *}$ & $-0.10,-0.02$ \\
\hline BMI $\left(\mathrm{kg} / \mathrm{m}^{2}\right)$ & + & & $-0.07^{\star \star}$ & $-0.12,-0.02$ & $t$ & & $0.07^{\star *}$ & $0.02,0.11$ \\
\hline \multicolumn{9}{|l|}{ Paternal } \\
\hline \multicolumn{9}{|l|}{ Educational level } \\
\hline High $v$. medium & $-0 \cdot 08^{\star \star}$ & $-0.13,-0.02$ & $0 \cdot 14^{\star \star \star}$ & $0.09,0.18$ & $t$ & & $-0 \cdot 08^{\star \star}$ & $-0.12,-0.03$ \\
\hline Low v. medium & 0.02 & $-0.03,0.07$ & 0.02 & $-0.03,0.07$ & $t$ & & 0.00 & $-0.05,0.06$ \\
\hline BMI $\left(\mathrm{kg} / \mathrm{m}^{2}\right)$ & $0 \cdot 05^{\star}$ & $0.00,0.09$ & + & & $-0 \cdot 06^{\star \star}$ & $-0.11,-0.02$ & $0.09^{\star \star \star}$ & $0.04,0.13$ \\
\hline
\end{tabular}

$\beta$, standardized regression coefficient; NL, Netherlands.

Results of backward regression analyses. All analyses were adjusted for recruitment group (alternative $v$. conventional lifestyle). Variables excluded in all four final models: child BMI Z-score and activity style; father's working hours and country of birth.

Pattern 1 = 'sedentary-snacking pattern'; pattern 2 = 'healthy intake pattern'; pattern 3 = 'sandwich pattern'; pattern 4 = 'sporty-traditional meal pattern'.

${ }^{\star} P<0.05 ;{ }^{\star \star} P<0.01 ;{ }^{\star \star \star} P<0.001$.

+Variable not included in the final model.

Table 4 Association of pattern scores with longitudinal BMI and weight status: KOALA Birth Cohort Study, The Netherlands

\begin{tabular}{|c|c|c|c|c|c|c|c|}
\hline & \multicolumn{4}{|c|}{ BMI Z-score } & \multicolumn{3}{|c|}{ Overweight } \\
\hline & \multicolumn{2}{|c|}{7 years } & \multicolumn{2}{|c|}{8 years } & \multicolumn{2}{|c|}{7 years } & \multirow{2}{*}{8 years } \\
\hline & $\beta$ & $95 \% \mathrm{Cl}$ & $\beta$ & $95 \% \mathrm{Cl}$ & OR & $95 \% \mathrm{Cl}$ & \\
\hline \multicolumn{8}{|l|}{ Pattern 1} \\
\hline Tertile 1 & 0.027 & $-0.019,0.073$ & 0.009 & $-0.041,0.059$ & 1.055 & $0.548,2.030$ & 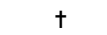 \\
\hline Tertile 3 & $0 \cdot 110^{\star \star \star}$ & $0.063,0.156$ & $0.089^{\star \star \star}$ & $0 \cdot 040,0 \cdot 138$ & $2 \cdot 222^{\star *}$ & $1 \cdot 230,4 \cdot 015$ & + \\
\hline \multicolumn{8}{|l|}{ Pattern 2} \\
\hline Tertile 1 & $t$ & & $t$ & & $\begin{array}{l}t \\
-\end{array}$ & & $\begin{array}{l}t \\
-\end{array}$ \\
\hline Tertile 3 & $\begin{array}{l}\dagger \\
-\end{array}$ & & $t$ & & $\begin{array}{l}t \\
t\end{array}$ & & $\begin{array}{l}t \\
t\end{array}$ \\
\hline \multicolumn{8}{|l|}{ Pattern 3} \\
\hline Tertile 1 & $t$ & & $\begin{array}{l}t \\
t\end{array}$ & & $\begin{array}{l}t \\
-\end{array}$ & & $\begin{array}{l}t \\
\text { r }\end{array}$ \\
\hline Tertile 3 & $\begin{array}{l}\dagger \\
-\end{array}$ & & $t$ & & $t$ & & 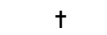 \\
\hline \multicolumn{8}{|l|}{ Pattern 4} \\
\hline Tertile 1 & $\dagger$ & & $\begin{array}{l}\dagger \\
-\end{array}$ & & $t$ & & $\begin{array}{l}t \\
t\end{array}$ \\
\hline Tertile 3 & + & & $t$ & & $t$ & & + \\
\hline
\end{tabular}

$\beta$, standardized regression coefficient.

Results of backward regression analyses. All analyses were adjusted for recruitment group; child gender, BMI Z-score at age 5 years, general appetite and activity style; parental educational level, working hours, country of birth and BMI.

Pattern 1 = 'sedentary-snacking pattern'; pattern 2 = 'healthy intake pattern'; pattern 3 = 'sandwich pattern'; pattern 4 = 'sporty-traditional meal pattern'.

${ }^{\star} P<0.05 ;{ }^{\star \star} P<0.01 ;{ }^{\star * \star} P<0.001$.

†Variable not included in the final model.

and intake of energy-dense foods are both important risk factors for childhood obesity ${ }^{(4)}$, putting children who score high on this pattern at a double risk for developing overweight or obesity. This is reflected in the positive association between this pattern and subsequent BMI and overweight development we found, in line with previous research $^{(23)}$. The fact that many previous studies $(22,24,27,29)$ did not find such an association between behavioural patterns and weight status can probably be attributed to the cross-sectional design of those studies. We also failed to find such a cross-sectional association, but the current study is one of the first to examine the longitudinal association of energy balance-related behavioural patterns with weight status in young children.

The second common pattern, the healthy intake pattern, included high intakes of mostly healthful foods such as fruit, vegetables, legumes and fish. Various studies have reported a similar pattern ${ }^{(9,10,13-16,18,22,25)}$, sometimes with the additional inclusion of high levels of $\mathrm{PA}^{(23-26,28)}$ and/or low levels of sedentary behaviour ${ }^{(24,28)}$. It is not clear why 
the PA behaviours in the current study did not cluster with the healthy intake pattern. A possible explanation could lie in the subdivision of PA into different PA types (e.g. active transport, sports at a sports club). However, secondary PCA with a sum score of all PA instead of the subtypes showed that this was not the case: the sum score of PA did not cluster with any of the patterns, while several of the subcomponents in the original analysis (i.e. school sports and sports at a sports club) did. An alternative explanation might lie in the make-up the KOALA cohort study. Findings of a previous clustering study in the KOALA cohort at age 2 years are in line with this explanation, as that study also revealed a healthy intake pattern without the inclusion of PA in that pattern ${ }^{(22)}$. Due to choice of recruitment methods, relatively many $(17 \cdot 9 \%)$ parents with an alternative lifestyle were included in the cohort. The majority of these alternative respondents have an explicit preference for healthy nutrition (e.g. vegetarian, organic ${ }^{(30)}$ ), while they do not have specific pronounced opinions regarding PA, as far as we know. The fact that recruitment channel was found to be a significant positive predictor of the healthy intake pattern score $(\beta=0 \cdot 24 ; P<0 \cdot 001)$ supports this hypothesis. Alternatively, PA might actually just not be associated with healthy dietary intake in the current population. Finally, a lower variability of certain variables in our population (compared with others) might limit the detection of such an association in the PCA.

The two other behavioural patterns found in the current study were the sandwich pattern and the sporty-traditional meal pattern. Although content-wise these patterns have no 'universal' counterparts in other studies, various studies do report the existence of a 'traditional' pattern ${ }^{(9,10,17,18,42)}$, of which the content is dependent on the country or region in which the study was conducted. Examples of such traditional patterns are a traditional Finnish pattern (rye, potatoes, milk, butter ${ }^{(9)}$ ), a British pattern (meat, potatoes $^{(10,17,18)}$ ) and a Mediterranean pattern (plant foods, oil, high eating frequency $\left.{ }^{(42)}\right)$. The traditional Dutch dinner ${ }^{(43)}$ is reflected in the sporty-traditional meal pattern, with a high intake of meat and potatoes. In a way, the sandwich pattern is also a traditional Dutch pattern, as the typical Dutch breakfast and lunch consist of sandwiches ${ }^{(44)}$. From an energy balance perspective, the two traditional patterns in the current study are considered neither healthy nor unhealthy, which is confirmed by the lack of an association between the pattern scores and longitudinal BMI and overweight development.

Various child characteristics were significantly associated with their pattern scores. Boys scored higher than girls on the sedentary-snacking and sandwich patterns. This adds to previous research showing that boys more often have an unhealthy intake pattern ${ }^{(15,19)}$. In addition, children with a large general appetite scored higher on both the healthy intake pattern and the sandwich pattern, while there was no significant relationship with the sedentary-snacking and sporty-traditional meal patterns.
This is an interesting finding, because it indicates that children with a larger appetite do not necessarily show less healthy behaviour. Various measures of impaired appetite regulation (e.g. low satiety responsiveness, high external food cue responsiveness) have previously been linked to childhood overweight and obesity ${ }^{(45)}$. However, a large appetite does not necessarily imply an impaired appetite regulation; children with a relatively large appetite might actually need more energy, and apparently get this from fairly healthy sources such as fruit and sandwiches. Parents can guide these children towards such healthy intake behaviours using specific parenting practices adapted to their child's eating style ${ }^{(46)}$. An alternative explanation is that parents might associate general appetite with intake during the main meals, and not with snacking behaviour.

In line with previous research ${ }^{(9,14,22)}$, scores on the healthy intake pattern were positively correlated with father's educational level, and scores on the sedentarysnacking pattern were inversely associated with parental educational level and positively associated with father's BMI. An explanation for these associations could lie in the possible mediating role of parenting practices. Mothers with a lower educational level or a higher BMI have previously been found to use less stimulation of healthy intake and $\mathrm{PA}^{(46)}$. Maternal working hours were inversely associated with the 'Dutch-traditional' sandwich and sporty-traditional meal patterns. Households that have a non-traditional parental role division (i.e. the mother also works) are possibly also less traditional in their eating patterns. Alternatively, working mothers might have less time to prepare traditional meals.

The current study has some limitations. All data, including dietary intake, activity behaviour and anthropometric data, were self-reported by the parents, which may have led to bias. Some have suggested the use of accelerometry instead of self-report data in studies examining clustering of activity behaviours in children ${ }^{(47)}$. However, although accelerometer data provide objective data regarding the intensity and duration of activities, they often cannot distinguish between different activity types ${ }^{(48)}$ and are thus unsuitable for the current study. The need to distinguish between different activity types is confirmed by the finding that different activity types cluster differently with the various behavioural patterns. The questionnaire used to assess activity behaviour in the current study, although standardized and widely used in The Netherlands, has not been validated previously. Furthermore, caution is warranted when generalizing our results, as there was an over-representation of 'alternative ${ }^{,(30)}$, relatively healthy lifestyle families in our sample. However, all regression analyses were all adjusted for recruitment channel. A final limitation lies in the choice of analytical methods. Both PCA, used in the current study, and cluster analysis rely on various subjective choices that may influence the outcomes. One of those subjective choices to 
be made is the cut-off point for component loadings. In the current study we chose a cut-off point of $0 \cdot 4$, in line with recommendations ${ }^{(38)}$, although cut-off points in previous studies varied from $0 \cdot 2$ to $0 \cdot 6$. Various behavioural items (including the items that were now not included in any of the patterns) would have been included in one of the patterns if a lower cut-off point had been used in the current study (e.g. television viewing for the sportytraditional meal pattern).

\section{Conclusions}

The current study found clustering of energy balancerelated behaviours, within the behavioural categories of activity behaviour and dietary intake (i.e. the healthy intake and sandwich patterns), as well as across these categories (i.e. the sedentary-snacking and sporty-traditional meal patterns). The sedentary-snacking pattern was positively related to longitudinal BMI and overweight development up to age 8 years. Furthermore, various parental and child background characteristics were associated with these patterns, providing indications for target groups for future childhood obesity prevention interventions. Especially children of parents with a lower educational level or a higher BMI seem important target groups. Such interventions should aim at targeting both dietary intake and activity behaviours simultaneously, thereby utilizing the synergy between activity behaviours and dietary intake.

\section{Acknowledgements}

The collection of data for the study was financially supported by the Dutch Ministry of Economic Affairs, the Dutch Ministry of Health, Welfare and Sports, FrieslandCampina and the Dutch Sugar Bureau, all in The Netherlands. The postdoctoral position of J.S.G. was financed by the NUTRIM School for Nutrition, Toxicology and Metabolism and the Faculty of Health, Medicine and Life Sciences, Maastricht University. The sponsors had no influence on the analysis and reporting of the study. None of the authors have any conflicts of interest to declare. All authors made substantial contributions to the design of the study. J.S.G., R.A.G., A.S. and C.T. were involved in the acquisition of the data. J.S.G. analysed and interpreted the data, and wrote draft versions of the manuscript. S.P.J.K. contributed to the interpretation of the data and the writing of the manuscript. All authors were involved in critically revising the manuscript, and have given their approval for the submitted manuscript.

\section{References}

1. World Health Organization (2004) Obesity: Preventing and Managing the Global Epidemic. Report on a WHO Consultation. WHO Technical Report Series no. 894. Geneva: WHO.
2. World Health Organization (2010) Childhood overweight and obesity on the rise. http://www.who.int/dietphysicalactivity/ childhood/en/ (accessed May 2011).

3. Singh AS, Mulder C, Twisk JW et al. (2008) Tracking of childhood overweight into adulthood: a systematic review of the literature. Obes Rev 9, 474-488.

4. Rennie KL, Johnson L \& Jebb SA (2005) Behavioural determinants of obesity. Best Pract Res Clin Endocrinol Metab 19, 343-358.

5. Schuit AJ, van Loon AJ, Tijhuis M et al. (2002) Clustering of lifestyle risk factors in a general adult population. Prev Med 35, 219-224.

6. Kremers SPJ, De Bruin G-J, Schaalma H et al. (2004) Clustering of energy balance-related behaviours and their intrapersonal determinants. Psychol Health 19, 595-606.

7. Reilly JJ, Jackson DM, Montgomery C et al. (2004) Total energy expenditure and physical activity in young Scottish children: mixed longitudinal study. Lancet 363, 211-212.

8. Kelder SH, Perry CL, Klepp KI et al. (1994) Longitudinal tracking of adolescent smoking, physical activity, and food choice behaviors. Am J Public Health 84, 1121-1126.

9. Mikkila V, Rasanen L, Raitakari OT et al. (2005) Consistent dietary patterns identified from childhood to adulthood: the cardiovascular risk in Young Finns Study. BrJ Nutr 93, 923-931.

10. Northstone K \& Emmett PM (2008) Are dietary patterns stable throughout early and mid-childhood? A birth cohort study. Br J Nutr 100, 1069-1076.

11. Hu FB, Rimm E, Smith-Warner SA et al. (1999) Reproducibility and validity of dietary patterns assessed with a foodfrequency questionnaire. Am J Clin Nutr 69, 243-249.

12. Millen BE, Quatromoni PA, Copenhafer DL et al. (2001) Validation of a dietary pattern approach for evaluating nutritional risk: the Framingham Nutrition Studies. $J$ Am Diet Assoc 101, 187-194.

13. Ritchie LD, Spector P, Stevens MJ et al. (2007) Dietary patterns in adolescence are related to adiposity in young adulthood in black and white females. J Nutr 137, 399-406.

14. Aranceta J, Perez-Rodrigo C, Ribas L et al. (2003) Sociodemographic and lifestyle determinants of food patterns in Spanish children and adolescents: the enKid study. Eur J Clin Nutr 57, Suppl. 1, S40-S44.

15. McNaughton SA, Ball K, Mishra GD et al. (2008) Dietary patterns of adolescents and risk of obesity and hypertension. J Nutr 138, 364-370.

16. Shin KO, Oh SY \& Park HS (2007) Empirically derived major dietary patterns and their associations with overweight in Korean preschool children. Br J Nutr 98, 416-421.

17. Northstone K \& Emmett P (2005) Multivariate analysis of diet in children at four and seven years of age and associations with socio-demographic characteristics. Eur J Clin Nutr 59, 751-760.

18. Pryer JA \& Rogers S (2009) Dietary patterns among a national sample of British children aged 1 1/2-4 1/2 years. Public Health Nutr 12, 957-966.

19. Rasanen M, Lehtinen JC, Niinikoski H et al. (2002) Dietary patterns and nutrient intakes of 7-year-old children taking part in an atherosclerosis prevention project in Finland. J Am Diet Assoc 102, 518-524.

20. Jago R, Fox KR, Page AS et al. (2011) Physical activity and sedentary behaviour typologies of 10-11 year olds. Int J Behav Nutr Phys Act 7, 59.

21. te Velde SJ, De Bourdeaudhuij I, Thorsdottir I et al. (2007) Patterns in sedentary and exercise behaviors and associations with overweight in 9-14-year-old boys and girls - a cross-sectional study. BMC Public Health 7, 16.

22. Gubbels JS, Kremers SP, Stafleu A et al. (2009) Clustering of dietary intake and sedentary behavior in 2-year-old children. J Pediatr 155, 194-198. 
23. Lioret S, Touvier M, Lafay L et al. (2008) Dietary and physical activity patterns in French children are related to overweight and socioeconomic status. J Nutr 138, 101-107.

24. Cameron AJ, Crawford DA, Salmon J et al. (2011) Clustering of obesity-related risk behaviors in children and their mothers. Ann Epidemiol 21, 95-102.

25. Yannakoulia M, Ntalla I, Papoutsakis C et al. (2011) Consumption of vegetables, cooked meals, and eating dinner is negatively associated with overweight status in children. J Pediatr 157, 815-820.

26. Yen LL, Chiu CJ, Wu WC et al. (2006) Aggregation of health behaviors among fourth graders in northern Taiwan. J Adolesc Health 39, 435-442.

27. Seghers J \& Rutten C (2011) Clustering of multiple lifestyle behaviours and its relationship with weight status and cardiorespiratory fitness in a sample of Flemish 11- to 12year-olds. Public Health Nutr 13, 1838-1846.

28. van der Sluis ME, Lien N, Twisk JW et al. (2011) Longitudinal associations of energy balance-related behaviours and crosssectional associations of clusters and body mass index in Norwegian adolescents. Public Health Nutr 13, 1716-1721.

29. Sabbe D, De Bourdeaudhuij I, Legiest E et al. (2008) A cluster-analytical approach towards physical activity and eating habits among 10-year-old children. Health Educ Res 23, 753-762.

30. Kummeling I, Thijs C, Penders J et al. (2005) Etiology of atopy in infancy: the KOALA Birth Cohort Study. Pediatr Allergy Immunol 16, 679-684.

31. Monitor Jeugdgezondheid (2009) Standaard Vraagstelling Bewegen (Standard Questionnaire Physical Activity). http://www.monitorgezondheid.nl/jeugdindicatoren.aspx (accessed August 2011).

32. Dutman AE, Stafleu A, Kruizinga A et al. (2010) Validation of an FFQ and options for data processing using the doubly labelled water method in children. Public Health Nutr (Epublication ahead of print version).

33. Stichting Nederlands Voedingsstoffenbestand (2001) Nederlands voedingsstoffenbestand 2001 (Netherlands Food Composition Table 2001). Zeist: Stichting Nederlands Voedingsstoffenbestand.

34. Fredriks AM, van Buuren S, Wit JM et al. (2000) Body index measurements in 1996-7 compared with 1980. Arch Dis Child 82, 107-112.

35. Barlow SE (2007) Expert committee recommendations regarding the prevention, assessment, and treatment of child and adolescent overweight and obesity: summary report. Pediatrics 120, Suppl. 4, S164-S192.

36. Eurostat (2007) Task Force on Core Social Variables. Final Report. Luxembourg: Office for Official Publications of the European Communities.

37. Field A (2005) Discovering Statistics Using SPSS. London: SAGE Publications.

38. Stevens JP (1992) Applied Multivariate Statistics for the Social Sciences. Hillsdale, NJ: Erlbaum.

39. Halford JC, Boyland EJ, Hughes G et al. (2007) Beyond-brand effect of television (TV) food advertisement/commercials on caloric intake and food choice of 5-7-year-old children. Appetite 49, 263-267.

40. Coon KA, Goldberg J, Rogers BL et al. (2001) Relationships between use of television during meals and children's food consumption patterns. Pediatrics 107, E7.

41. Temple JL, Giacomelli AM, Kent KM et al. (2007) Television watching increases motivated responding for food and energy intake in children. Am J Clin Nutr 85, 355-361.

42. Kontogianni MD, Farmaki A-E, Vidra N et al. (2010) Associations between lifestyle patterns and body mass index in a sample of Greek children and adolescents. $J$ Am Diet Assoc 110, 215-221.

43. van Dam RM, Grievink L, Ocke MC et al. (2003) Patterns of food consumption and risk factors for cardiovascular disease in the general Dutch population. Am J Clin Nutr 77, 1156-1163.

44. Raaijmakers LG, Bessems KM, Kremers SP et al. (2011) Breakfast consumption among children and adolescents in the Netherlands. Eur J Public Health 20, 318-324.

45. Carnell S \& Wardle J (2008) Appetitive traits and child obesity: measurement, origins and implications for intervention. Proc Nutr Soc 67, 343-355.

46. Gubbels JS, Kremers SP, Stafleu A et al. (2011) Association between parenting practices and children's dietary intake, activity behavior and development of body mass index: the KOALA Birth Cohort Study. Int J Behav Nutr Phys Act 8, 18.

47. Saunders TJ, Prince SA \& Tremblay MS (2011) Clustering of children's activity behaviour: the use of self-report versus direct measures. Int J Behav Nutr Phys Act 8, 48.

48. Jago R, Fox KR, Page AS et al. (2011) Physical activity and sedentary behaviour typologies of 10-11 year olds response to Saunders and colleagues. Int J Behav Nutr Phys Act 8, 49. 